\title{
Fear Filter: Visualising the UK terror threat level
}

\author{
Rod Dickinson \\ University of the West of England \\ Bristol, UK \\ Rod.dickinson@uwe.ac.uk
}

\begin{abstract}
Fear Filter is a digital artwork comprising of a mobile phone photo filters application for Android / iOS platforms and a digital photo stream. The photo filters are created by gathering current and historical information about the UK Threat Level from a live feed from MI5, the UK security service. Photographs taken with the mobile application are transformed by the photo filters, each of which correspond to a different moment in time and the related Threat Level from that period. The filters cover the period 1 August 2006 until the present moment. Photos shared from the mobile application are automatically posted to a public photo stream. The artwork draws together the ubiquity of photography as a networked medium and the growing use of photo filters apps with the invisible ubiquity of the UK Threat Level. Utilising pattern recognition and algorithmic image analysis each photo filter visualises the UK Threat Level by colourising and redacting the subject of the photograph according to the severity of the Terror Threat Level. Fear Filter exploits the confluence of mobile digital photography, platforms, networks and the online security theatre of the UK Threat Level to reformulate the relationship between photography and terrorism.
\end{abstract}

Algorithmic image. Art. Information. Digital platforms. Fear. Networks. Photography. Security theatre. Terror. Terrorism.

\section{INTRODUCTION}

Fear Filter is a digital artwork comprising of a mobile phone photo filters application for Android / iOS platforms and a digital photo stream. The photo filters are created by gathering current and historical information about the UK Terror Threat Level from MI5, the UK security service. Photographs taken with the mobile application are transformed by the photo filters, each of which correspond to a different moment in time and the related Terror Threat Level from that period.

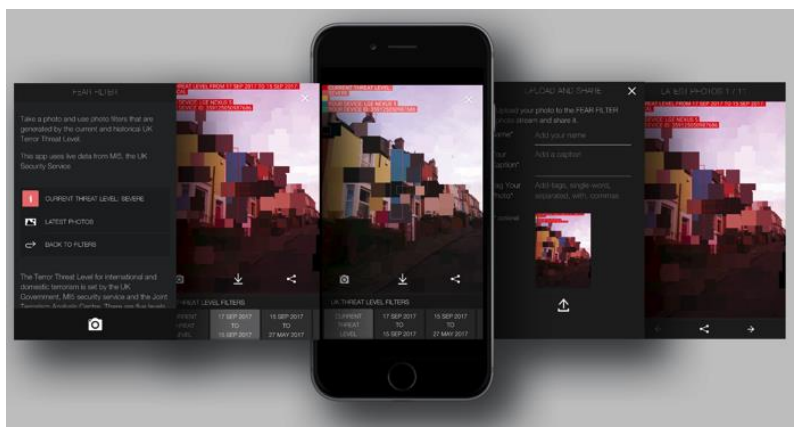

Figure 1: Fear Filter mobile app screens and filters.
Images can be captioned and tagged and shared from the app via the digital photostream that collates all the photos on a publicly accessible web platform (www.fearfilter.uk).

Fear Filter utilises many of the features of a platform, a digital infrastructure that combines digital tools to enable users to produce and distribute a service or a digital artefact (a photograph). Fear Filter uses a digital platform as medium and typical of most digital platforms manipulating data and user data is central to its operations, (Srnicek 2017).

\section{THE UK THREAT LEVEL}

Since 1970, the UK government has used a system of alerts as a method to communicate the assessed threat from terrorism and military threats (Select Committee on Defence, UK Government 2003). Now called the UK Threat Level it was initially only displayed in government buildings and institutions, but since 2006 it has been published and publicly displayed on the home office and MI5 web sites (Select Committee on Defence 2003, MI5 Security Service 2018). 
In both iterations, from 1970-2006 and from 2006 onward the Threat Level has taken the same form: A series of escalating, named levels and corresponding colours which currently mimic the generic green to amber to red colour schemes of many warning systems. From 2006 the threat level has explicitly referred to the likelihood of a terror related attack. Prior to 2006 the obtusely named Bikini State system additionally referred to military threats. It used idiosyncratically named levels such as Bikini Black Special, which have now been replaced by more accessible names: Low, Moderate, Substantial, Severe and Critical. The current Threat Level is also published as a live, public RSS feed used by news outlets and government organisations. The Fear Filter mobile phone application hijacks the RSS feed to create a filter that responds to changes in the Threat Level in real time, alongside using historical information to generate a suite of swipeable filters that represent each Threat Level since 1970 and plot the Threat Level over time.

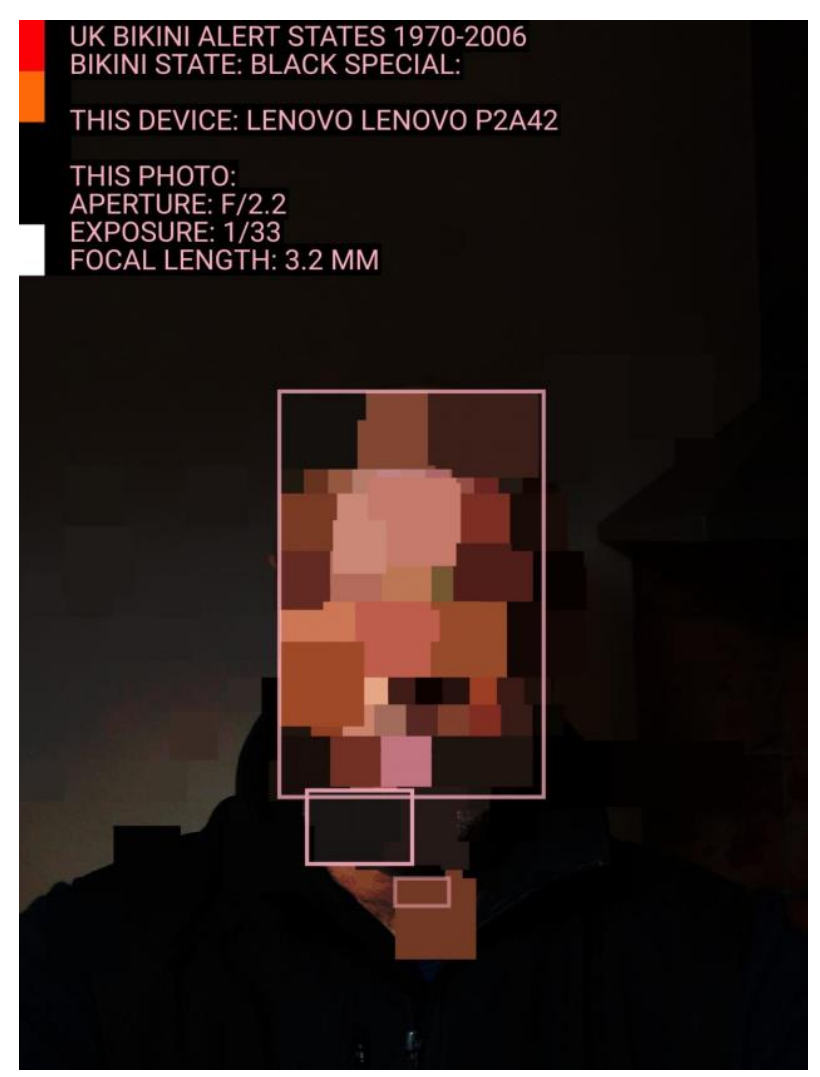

Figure 2: Sample image produced by the Fear Filter mobile phone application.

The mobile phone application uses the Threat Level to define a set of colour tints for the filters, based on home office defined colours for each Level. The filters also redact a percentage of the image by pixelating it using a sliding scale where the severity of the threat correlates to the area of redaction. The higher the Threat Level the greater the percentage of redaction. Each image is also subject to a basic computer vision algorithm that identifies colours and objects in the photographic frame and targets them for redaction. Finally, metadata is printed onto each image. This includes EXIF data from the photograph; the location, aperture and other photographic specific information, but it also includes particular information about the Threat Level associated with each filter.

The filters are arranged as a timeline, each filter demarcates a period of time and is associated with a specific Threat Level for that period. When used the filter applies the colours and level of redaction associated with that Threat Level, allowing the user to see (and apply) the Threat Level back to 1 August 2006.

\begin{tabular}{|c|c|c|}
\hline \multicolumn{3}{|c|}{ FILTERS : UK THREAT LEVEL OVER TIME } \\
\hline CURRENT & 1 MAR 2018 & 15 SEP 2017 \\
\hline THREAT & TO & TO \\
\hline LEVEL & 15 SEP 2017 & 17 SEP 2017 \\
\hline 27 MAY 2017 & 23 MAY 2017 & 11 MAY 2016 \\
\hline TO & TO & TO \\
\hline 15 SEP 2017 & 27 MAY 2017 & 23 MAY 2017 \\
\hline 29 AUG 2014 & 24 OCT 2012 & 11 JUL 2011 \\
\hline TO & TO & TO \\
\hline 11 MAY 2016 & 29 AUG 2014 & 24 OCT 2012 \\
\hline 24 SEP 2010 & 22 JAN 2010 & 20 JUL 2009 \\
\hline TO & TO & TO \\
\hline 11 JUL 2011 & 24 SEP 2010 & 22 JAN 2010 \\
\hline 4 JUL 2007 & 30 JUN 2007 & 13 AUG 2006 \\
\hline TO & TO & TO \\
\hline 20 JUL 2009 & 4 JUL 2007 & 30 JUN 2007 \\
\hline 10 AUG 2006 & 1 AUG 2006 & BACK \\
\hline TO & TO & TO \\
\hline 13 AUG 2006 & 10 AUG 2006 & ORIGINAL \\
\hline
\end{tabular}

Figure 3: Threat Level Timeline: Each filter in the mobile app demarcates a period of time and is associated with a specific Threat Level for that period.

\section{FEAR AS GOVERNANCE}

In his discussion on security and terror Giorgio Agamben argues that since the birth of the modern state security has always been at the core of state politics and is the basic principle of state activity (Agamben 2009).

The UK Threat Level can be thought of as a component of state security: On their website MI5 write that the UK national security strategy directs and guides their policies (MI5 Security Service 2018). In many ways Agamben stands at the end of a long line of thinkers that have considered the relationship between security and fear. Thomas 
Hobbes' seminal "contract" (1651) derives directly from his equation between fear and security: The fear that a civilisation without a "common power" (Hobbes 1651) or ruler to keep all men "in awe" (Hobbes 1651) will result in a life for everyone that has no security and is a constant state of war, with "every man against every man" (Hobbes 1651). Hobbes' vision was that subjects should cede freedoms to the ruler to be protected from a life that would otherwise be "solitary, poor, nasty, brutish, and short" (Hobbes 1651, Ludlow 2014).

For Hobbes and others security is ensured by the threat of violence and the fear that attends it, either from the sovereign or from other citizens (Hobbes 1651, Weber 2009). However, others propose that rather than being a by-product of the threat of violence, fear is a political tool where leaders, governments or even militants dictate what should be the object of fear and use it to dominate political agendas (Ludlow 2014, Robin 2004). In this model, the object of fear is often directed at a designated other, often an external force (Svendsen 2008) or groups perceived as other, as typified by the ongoing War on Terror.

The impossible practicalities of providing security in this context, against what George W Bush described as a threat "to the very idea of civilised society" (Svendsen 2008) is encapsulated by Brian Holmes, who, describing the difficulty and absurdity of making secure a whole society contends that the result is "vertiginous paranoia" and a "security panic" that produces a proliferation of "eyes, ears, cameras, snooping devices, data banks, crosschecks and spiralling analytical anxiety" (Holmes 2009).

In less hysterical terms Louise Amoore describes this phenomenon (also referring to the US) as "watchful politics" (Amoore 2007). Both Holmes and Amoore cite the rapidly growing use of image and data surveillance techniques, including the interweaving of digital and image based technologies that utilise facial and gait recognition software and biometric identity cards and passports (now common in the UK). Although initially predating the War on Terror the UK Threat Level belongs to this explosion of security apparatus: In 2006, a year after the 7/7 London tube bombing, the Threat Level was revamped with less obtuse sounding Threat Level names (for example, the bizarrely named "Bikini Black Special" Threat Level was replaced with the much simpler "Severe") and for the first time the current Threat Level was publicised and published (MI5 Security Service 2018).

\section{SECURITY THEATRE}

The UK Threat Level belongs to a suite of relatively recent counter terror measures where their symbolic presence and visibility seems more important than actually improving security (Schneier 2009). Despite the visibility of the Threat Level in the national media advice on how to respond to it is vague and contradictory: MI5 suggest that "Threat levels in themselves do not require specific responses from the public" (MI5 Security Service 2018), even though they go on to suggest the public should be vigilant at all times.

Many of the prominent security measures that we are now familiar with, such as the ban on carrying liquids through airport security, full body scans at airports (Levenson 2018), the many instances of harassment of photographers (Vallée 2009) alongside the Threat Level might be considered to be examples of Security Theatre. Where in each of these cases the appearance or illusion of security far outweighs the effect in actually increasing security or reducing the risk of a terrorist attack. Security Theatre might partly be seen as a counter spectacle to the symbolic character of much contemporary terrorism, where the primary aim is to produce the rhetorical visibility of security. The filters in the Fear Filter app show how the UK Threat Level has changed over time, and they reveal that since 2006 it has only been lowered from Severe three times. So, despite being presented as a way of making us secure the UK Threat Level might instead simply remind us to be fearful, or even more likely, make us acutely aware of its theatricality.

\section{NETWORKED PHOTOGRAPHY}

At around the time that the Threat Level was first publicised and published photography started to make increasing use of network technologies, initially with the emergence of digital phone cameras and online photo hosting websites. Since then contemporary photography has been increasingly defined as a socio-technical network: An assemblage of technologies, networks and human endeavour (Gómez Cruz \& Meyer 2012).

For much of the early digital age many scholars focused more on the malleability of the digital image, rather than its distribution and entanglement with digital networks. In particular the ease with which photographs could be altered, thereby potentially undermining any claim of their evidential status (Mitchell 1994). In recent years this has changed with a greater focus on the emergence of practices such as citizen journalism, which in turn are facilitated by the increased sophistication of camera phone technology, mobile internet and the 
platforms and apps that have developed to take, edit, host and share images seamlessly (Gómez Cruz \& Meyer 2012, Hand 2012, Rubinstein, Golding \& Fisher 2013). Well known current examples include Flickr, Instagram and Photobucket. The immediacy and speed of transmission across the network has now become an important measure of the currency of a photo (Rubinstein \& Sluis 2008) even at the expense of the quality of the image or concerns about its veracity: Describing an early live TV broadcast from the initial stages of the 2003 invasion of Iraq artist Hito Steyerl compares the resultant blurry, low resolution image of green and brown blotches to a "military form of abstract expressionism" (Steyerl 2007) proposing that the more immediate the image the less legible it is. Similarly one of the most published photographs of the 2005 London $7 / 7$ underground bombing is a badly lit, low resolution camera phone image taken by Eliot Ward of his friend Adam Stacey on his way out of a tube carriage immediately after the bombing. Significantly Ward's image was distributed to media outlets within hours of the event after he had uploaded it to the photo blogging website moblog.co.uk using a Creative Commons licence (Rubinstein \& Sluis 2008; Wardle 2015).

A year earlier journalist Maureen Dowd (2004) had recorded Donald Rumsfeld's (United States Secretary of Defense from 2001 to 2006) reaction after seeing the now, well known, Abu Ghraib prisoner abuse photographs. His surprised comments encapsulate the new ease with which citizens (or in this case soldiers) could become photographer and distribution agency all in one:

\begin{abstract}
In the information age people are running around with digital cameras and taking these unbelievable photographs and then passing them off, against the law, to the media, to our surprise, when they had not even arrived in the Pentagon.
\end{abstract}

Increasingly networked photography is built into digital platforms that host, share, collate and distribute images. Fear Filter utilises many of the parameters and conventions of digital photo platforms such as Flickr, particularly the ability to share and distribute images to other platforms and networks directly from the mobile app.

The prevalence of the use of tagging and hashtags as a form of architecture and a method of browsing, moving from photo to photo and tag to tag (Cox 2008) is also replicated in Fear Filter. Following convention the tags in Fear Filter are user generated, user descriptions of their images. This user generated organisation creates a categorisation and navigation system (Rubinstein \& Sluis 2008) and much like Flickr and Instagram Fear Filter organises streams of photos under each tag, so that the experience of navigation is less like looking at an individual photo and more an experience of a sequence of images. Michelle Henning describes this experience as "flow" (Henning 2018), with all the associations of the friction free movement of goods or of energy that this implies. In Fear Filter as in other digital photo platforms the "flow" of the images would also be inconceivable without the mobile multi-touch interface that supports the physical gestures that constitute the sense of "flow".

\section{FEAR FILTER, PHOTOGRAPHY AND TERROR}

Fear Filter exploits the confluence of mobile digital photography, platforms, networks and the online security theatre of the UK Threat Level to reformulate the relationship between photography and terrorism.

Many of the most celebrated photographs of terrorism put victims, perpetrators, event or place within the frame of the image, producing what Henry Giroux calls "The Spectacle of terrorism" (Giroux 2007). Fear Filter uses a different methodology; superimposing a visualisation of the UK Threat Level onto all photographs irrespective of their subject, or of their framing. Rather than using the imagery associated with terror and terrorism Fear Filter uses a computational equation, a programmatic placeholder for the UK Terror Threat. The photo becomes a record of this pervasive form of security theatre, rather than a recorded image of terrorism. But perhaps this even more closely echoes James Der Derian assertion that "we see terrorism everywhere in real time, all the time" (Derian 2005). The ubiquitous network technologies that make this possible, mobile internet, live http streaming, mean terrorism has also taken on a "highly optical character" (Derian 2005) with images at the centre of the Spectacle of Terrorism and images at the centre of a set of technologies that are part of the "watchful politics" described by Amoore (2007). Fear Filter sits between these practices catching the points of intersection to transform everyday photography into a record of the contemporary tension between fear and security.

\section{REFERENCES}

Agamben, G., and Emcke, C. (2009) Security and terror. In Cox, G., and Sutzl, W. (eds.). Data browser 04 Creating Insecurity: Art And Culture In The Age Of Security, Autonomedia, Brooklyn, NY.

Amoore, L. (2007) Vigilant visualities: The watchful politics of the war on terror. Security Dialogue, 38(2), pp.215-232. 
Cox, A. M. (2008) Flickr: a case study of Web 2.0. Aslib Proceedings, 60(5), pp.493-516.

Der Derian, J. (2005) Imaging terror: logos, pathos and ethos. Third World Quarterly, 26(1), pp.23-37.

Dowd, M. (2004) A World Of Hurt. http://www.nytimes.com/2004/05/09/opinion/aworld-of-hurt.html (retrieved 16 March 2018).

Giroux, H. A. (2007) Beyond the spectacle of terrorism: rethinking politics in the society of the image. Situations: Project of the radical imagination, 2(1), pp.17-51.

Hand, M. (2012) Ubiquitous Photography. Polity, Cambridge UK.

Henning, M. (2018) Image Flow Photography on tap. Photographies, 11(2).

Hobbes, T. (1651) Leviathan.

Holmes, B. (2009) Security aesthetic = systems panic. In Cox, G. and Sutzl, W. (eds.). Data browser 04 Creating Insecurity: Art And Culture In The Age Of Security, Autonomedia, Brooklyn, NY.

Levenson, E. (2018) The TSA Is in the Business of 'Security Theater,' Not Security.

https://www.theatlantic.com/national/archive/2014/0 1/tsa-business-security-theater-notsecurity/357599/ (retrieved 12 March 2018).

Ludlow, P. (2014) Fifty States of Fear. The Stone. https://opinionator.blogs.nytimes.com/2014/01/19/fif ty-states-of-fear/ (retrieved 12 March 2018).

Steyerl, H. (2007) Documentary Uncertainty. A prior, 15, pp.300-308.

MI5 Security Service (2018) Threat Levels. https://www.mi5.gov.uk/threat-levels (retrieved 7 March 2018).

MI5 Security Service (2018) What We Do. https://www.mi5.gov.uk/what-we-do (retrieved 7 March 2018).
Mitchell, W. J. (1994) Intention and Artifice. In The Reconfigured Eye: Visual Truth In The PostPhotographic Era. MIT Press, Cambridge, MA.

Robin, C. (2004) Fear: The history of a political idea. Oxford University Press, New York.

Rubinstein, D., and Sluis, K. (2008) A Life More Photographic: Mapping the networked image. Photographies, 1(1), pp.9-28.

Rubinstein, D., Golding, J., and Fisher, A. (2013) On the Verge of Photography. Imaging Beyond Representation. ARTicle Press, Birmingham.

Schneier, B. (2009) Beyond Security Theater. https://www.schneier.com/essays/archives/2009/11 /beyond security thea.html (retrieved 12 March 2018).

Svendsen, L. (2008) A Philosophy of Fear. Reaktion Books, London.

Srnicek, N. (2017) Platform Capitalism. Polity, Cambridge UK.

Vallée, M. (2009) We're photographers, not terrorists.

https://www.theguardian.com/commentisfree/liberty central/2009/dec/11/photographers-section-44terrorism-act (retrieved 7 March 2018).

Wardle, C. (2015) 7/7: Comparing the use of eyewitness media 10 years on.

https://medium.com/1st-draft/7-7-comparing-theuse-of-eyewitness-media-10-years-ona4015e548cef (retrieved 12 March 2018).

Weber, M. (2009) Politics as a Vocation. In Max Weber: Essays in Sociology. Routledge, Oxon.

UK Government (2003) Select Committee on Defence Minutes of Evidence.

https://publications.parliament.uk/pa/cm200203/cm select/cmdfence/557/3032007.htm (retrieved 7 March 2018).

UK Government (2018) Terrorism and national emergencies. https://www.gov.uk/terrorismnational-emergency (retrieved 7 March 2018). 